\title{
Arthrocentesis of the temporomandibular joint: A review
}

\author{
Peter Tvrdy, Petr Heinz, Richard Pink
}

\begin{abstract}
Background. Arthrocentesis is a very gentle method for lavage of the joint space. The principle consists in the introduction of a pair of needles into the upper joint space and subsequent lavage using physiological saline or Ringer's solution. Arthrocentesis of the temporomandibular joint is used in both cases of acute closed lock and treatment of various temporomandibular disorders.

Methods. A literature search in Pubmed database, using key words: Temporomandibular joint (TMJ), Arthrocentesis, indications, technique, results.

Conclusion. Arthrocentesis of the temporomandibular joint is a minimally invasive treatment method at the boundary between conservative and surgical therapy. It is usually performed on an out-patient basis under local anaesthesia. It is used both in cases of acute block caused by displacement of the articular disc and also to treat degenerative inflammatory diseases of the joints. The main objective of arthrocentesis is to wash out inflammatory mediators, release the disc, break adhesions, eliminate pain and improve joint mobility. It is a method with a minimum number of complications, it is simple and not demanding in terms of instruments, and it can be performed repeatedly. For this reason, it has become widespread and very popular in the treatment of internal disorders of the temporomandibular joint.
\end{abstract}

Key words: arthrocentesis, temporomandibular joint

Received: November 8, 2012; Accepted: April 3, 2013; Available online: April 10, 2013

http://dx.doi.org/10.5507/bp.2013.026

Department of Oral and Maxillofacial Surgery, University Hospital Olomouc and Faculty of Medicine and Dentistry, Palacky University Olomouc, Czech Republic

Corresponding author: Petr Tvrdy, e-mail: peter.tvrdy@fnol.cz

\section{INTRODUCTION}

Temporomandibular disorders (TMD) represent a wide range of functional changes and pathological conditions affecting both the jaw joint itself and the chewing muscles and, ultimately, all the other components of the oromaxillofacial system. In recent years, TMD have become an increasingly frequent cause of seeking medical assistance. The number of patients is growing, probably due to the psychological tension in our society today. Temporomandibular disorders have a multifactorial pathogenetic background and a highly varied clinical picture. According to earlier accepted psychophysiological concepts, occlusal problems and emotional stress were considered to be the most serious aetiological factors. However, the causes of the development of TMD are likely to be far more complex. Based on a comprehensive understanding of the issue, it is, today, somewhat of a mistake to focus solely on the jaw joint. Instead, it is necessary to consider the entire chewing apparatus. Initial indistinct problems lead to the fact that patients do not present themselves until the symptoms have evolved and, in many cases, only in the period of irreversible morphological and functional changes. Therefore, greater attention should be paid to these disorders, their prevention, earlier detection, and timely treatment.

Characteristic symptoms of temporomandibular joint disorders are pain, changes in the mobility of the lower jaw (in the sense of restricted opening of the mouth hypomobility, or, by contrast, hypermobility), and sound phenomena (clicking, grinding). There are essentially two types of therapy for temporomandibular joint (TMJ) disorders - conservative (non-invasive) and surgical (invasive), during which the joint structures themselves are entered. Conservative treatment includes bite splints, rehabilitation exercises, isometric exercises, massage of the chewing muscles, analgesic treatment, thermotherapy or laser therapy. Surgical treatment can be divided into invasive (open) and minimally invasive (which includes arthrocentesis and arthroscopy) (ref. ${ }^{1}$ ).

\section{HISTORICAL}

The first detailed description of the indications, technique and therapeutic benefit of arthrocentesis dates back to 1592, when Fray Augustin Farfan described, in the second edition of his work Tractado breve de medicina in Mexico, a knee arthrocentesis. However, the first mention can be found in Libellus de medicinalibus Indorum herbis, known as the Codexus Badianus, dated 1552, which was a translation of an Aztec manual of herbal medicine for the Spanish king Charles V ( ref. $\left.^{2}\right)$.

Arthrocentesis of the temporomandibular joint was first described by D. W. Nitzan in 1991 as the simplest form of surgical therapy with the aim of washing out inflammatory mediators, releasing the articular disc, and disrupting adhesions between the surface of the disc and the joint fossa by hydraulic pressure of the lavage solution ${ }^{3}$. 


\section{DESCRIPTION OF THE PROCEDURE}

The principle consists in the introduction of a pair of needles into the upper joint space and subsequent lavage using a solution of physiological saline or Ringer's solution. One needle is used to introduce the solution into the joint, and the other one is used to drain the liquid. The upper joint space is best accessible through a skin puncture, which is located about $10 \mathrm{~mm}$ ventrally from the tragus, on an imaginary line connecting the tragus and the outer corner of the eye (the Holmlund line). This location corresponds to the maximum concavity of the glenoid fossa 4 . While the patient's mouth is open, the needle is inserted up, towards the front, and inwards, to a depth of about 2 $\mathrm{cm}$ after the tip of the needle has come into contact with the posterior wall of the articular eminence. After the first needle with a syringe containing $2 \mathrm{~mL}$ of physiological saline or Ringer's solution is inserted, the application is performed using gentle pressure. If approximately the same amount of fluid is subsequently aspirated back, it shows that the needle location in the upper joint space is correct. During the application, movement of the chin portion of the lower jaw in a contralateral direction can sometimes be noted. The second needle is inserted $10 \mathrm{~mm}$ anterior to the first one, $2 \mathrm{~mm}$ under the line connecting the tragus and the outer corner of the eye ${ }^{4}$.

The procedure is usually performed under local anaesthesia using auriculotemporal nerve block; however, there are 5 studies in which patients underwent arthrocentesis under general anaesthesia. At the same time, it was not specified whether this was the physician's decision or the patient's wish ${ }^{5}$.

After the introduction of both needles, a syringe with the mentioned solution is attached to the first one, and lavage of the joint space is performed. At the same time, the second needle acts as an outflow portal and allows drainage of the joint lavage material ${ }^{6}$. The volume of the lavage fluid mentioned in various published studies ranges between 50 and $500 \mathrm{~mL}$. The fibrous tissue of the articular disc has a better tolerance for Ringer's solution than for an isotonic saline solution ${ }^{6,7}$.

The amount of solution is important as well. In his study, Zardeneta recommends $100 \mathrm{~mL}$ of Ringer's solution, which is sufficient to eliminate specific proteins and proteases $^{8}$. In contrast, Kaneyama recommends 300-400 $\mathrm{mL}$, during which interleukin 6 , bradykinin and other proteins are washed out ${ }^{9}$.

As access to the anterior recess of the upper joint space is not needed as much as during arthroscopic visualization, the second needle can be introduced parallel, approximately 2-3 $\mathrm{mm}$ anterior to the first one. This method is technically less challenging and more comfortable for both the patient and the physician. Both needles are introduced into the wider part of the joint space; therefore, frequently, the lavage is more successful ${ }^{10}$.

Another option is to use a single-needle technique, which consists of introducing only one injection needle into the upper joint space. Compared with the traditional two-needle approach, this method has certain advantages. The procedure is shorter and gentler and it has fewer potential complications. There is no risk of damage to the facial nerve branches, which are located at the site of introduction of the second needle. The single-needle method allows for fluid injection into the joint space at a certain pressure, which achieves an enlargement of the joint space and severance of any adhesions inside the joint. The needle is introduced while the mouth is open. Next, the patient is asked to close his or her mouth. This way, the fluid is drained from the joint space through the same needle. The procedure is repeated at least ten times, and the total amount of fluid is $50 \mathrm{mLl}\left(\right.$ ref. ${ }^{11}$ ). Adhesions occurring inside the temporomandibular joint explain the phenomenon of adherence of the disc to the fossa or eminence. Their lysis leads, in most cases, to improved mouth opening. Therefore, the single-needle method is indicated in cases of joints with significant hypomobility and adhesions or in cases of joints with advanced degenerative changes that could greatly complicate the introduction of the second needle ${ }^{12}$.

Today, arthrocentesis of the temporomandibular joint is used not only in cases of acute closed lock but also in the treatment of various temporomandibular disorders. Thus, the most frequent indication is an acute anterior displacement of the articular disc without reduction or hypomobility of the joint with occurrences of disc adhesions (a stuck disc). It is possible to select arthrocentesis as a palliative procedure for patients with an acute episode of degenerative or rheumatoid arthritis and also for patients with a painful displacement of the disc with reduction, which rarely responds to conservative treatment. Treatment success is prominent in cases of acute patients or patients with a history of short-term problems ${ }^{13,14}$.

All studies that examine outcomes of arthrocentesis in the treatment of internal temporomandibular joint disorders contain a significant limitation - small sample size, the influence of concomitant use of anti-inflammatory drugs and analgesics and also the inability to generalize the results to a male population because a significant majority of patients with temporomandibular dysfunction are women ${ }^{10}$.

It is becoming evident that arthrocentesis with lavage of the joint space is a very good treatment method for inflammatory and degenerative diseases of the temporomandibular joint. The question is whether the results would be better if accessory intra-articular application of corticosteroids or hyaluronic acid were used. An application of the mentioned substances, which remain active in the joint space, is possible towards the end of arthrocentesis especially for degenerative disabilities in cases employing the single-needle technique. In a randomized blind study of 72 patients, Manfredini and Rancitelli failed to demonstrate any statistically significant difference between the group that underwent only arthrocentesis and lavage of the joint space and the group that received, at the end of the arthrocentesis, corticosteroids or hyaluronic acid ${ }^{15}$.

Lavage of the upper joint gap helps to reduce pain by removing inflammatory mediators from the joint. These are the different types of inflammatory and antiinflammatory cytokines, the balance of which affects the development of degenerative and inflammatory changes. 
Inflammatory cytokines include interleukin-1, interleukin-6, interleukin-8 and TNF-alfa while anti-inflammatory cytokines include interleukin-4, tissue inhibitors of metalloproteinases TIMP-1, TIMP-2 and tumour growth factor TGF-beta ${ }^{16}$. In patients with an internal disease, high levels of IL-6 in the synovial fluid of the temporomandibular joint are associated with extensive acute synovitis. In the course of the inflammation, monocytes and macrophages quickly release IL-1 as well as IL-6. Fibroblasts and chondrocytes also have this ability but at the same time, through the action of IL-6, they release TIMP as well. Further, IL-6 was detected in synovial cells and mononuclear cells infiltrating the edge of the blood vessels. These cells produce IL-6 in both synovial tissue and synovial fluid ${ }^{17}$.

Usually, a comparison is done of the levels of individual cytokines before and after minimally invasive therapy of internal disorders of the temporomandibular joint and osteoarthritis. Most of the literary sources mention comparative studies that evaluate arthrocentesis outcomes. In this case, the level of cytokines is assessed after 7 to 14 days, and in the case of pharmacological treatment of osteoarthritis, the concentration of cytokines is determined after 14 days and after 14 weeks. The results are shown as a percentage, or in picograms per millilitre (pg/ $\mathrm{mL})\left(\right.$ ref. ${ }^{18}$ ).

Most authors have present statistically significant differences in concentration of the monitored cytokines before and after arthrocentesis, in the sense that their levels were lowered. Arthrocentesis is considered to be successful if there is a decrease of the levels of IL-6, IL-8, IL-11 and TNF-alfa, which, however, are still detectable ${ }^{19}$.

The success rate of arthrocentesis mentioned in the literature ranges between $70-90 \%$ ( ref. $^{20,21}$ ). However, the question is whether mere arthrocentesis and lavage of the joint space can assist to reposition a displaced disc to its normal position. Literary sources have mentioned cases of disc reposition; however, the success rate depends on the duration of the displacement. The retrodiscal tissue becomes less elastic and more fibrous over time; therefore, it is more difficult to shift the articular disc. In many cases, arthrocentesis is not sufficient, and it is necessary to select a combined approach using mandibular manipulation, occlusal splints and rehabilitation. Nonetheless, full disc reposition is not necessary to restore good function of the joint and to alleviate the pain of patients with a closed lock ${ }^{22,23}$.

Arthrocentesis is also associated with some complications, the severity of which depends on the anatomy of the joint, its relation to the surrounding structures, and also the method used to introduce the needles. The frequency of these complications mentioned in the literature ranges between 2 and 10\%.

These complications include injury to the facial nerve, preauricular hematoma, injury to the superficial temporal artery, the development of arteriovenous fistula, bleeding into the joint, intracranial perforation, extradural hematoma, breakage of a part of the needle in the joint, unsuccessful introduction of the needle, leakage of the lavage fluid into the extra-articular space in the area, damage to the surface of joint, an allergic reaction to the anaesthetic or drugs that may be administered at the end of the arthrocentesis. In addition, an acute inflammation of the joint may occur, which may be accompanied by preauricular oedema, redness, pain, and restricted mouth opening. Moreover, otologic complications can be serious in connection with the close proximity of the temporomandibular joint, the middle ear, and the cartilaginous wall of the ear canal. This includes perforation of the external auditory canal, the occurrence of blood clots in the external auditory canal, tympanic membrane injuries, partial hearing loss, a feeling of a blocked ear and dizziness ${ }^{6}$.

An increased risk of facial nerve damage exists when repeatedly attempting to introduce a needle into the joint space after an unsuccessful primary needle insertion. In such cases, the single-needle method appears to be very gentle, as mentioned above ${ }^{24,25}$.

The bone of the glenoid fossa is part of the base of the skull. Its thickness is in the range of 0.5 to $1.5 \mathrm{~mm}$. During arthrocentesis or arthroscopy, the bone may be perforated, and subsequently, intracranial needle penetration may occur. The surgeon must be very careful during the needle introduction and must correct the pressure on the needle during its insertion.

Extradural hematoma has been described by Caroll et al. ${ }^{26}$, and preauricular infectious oedema has been described by Nitzan $^{27}$. Oedema caused by leakage of the lavage fluid (Ringer's solution) into the extra-articular soft tissues usually resolves within a day.

An inflammatory focus (abscess or cellulitis) at the site of the needle insertion during arthrocentesis is generally considered an absolute contraindication for this procedure. Bacteraemia, adjacent osteomyelitis, coagulopathy, and a malignant tumour are considered as relative contraindications. In a prospective study conducted by Thumboo and Duffy using a sample of 32 patients who were on the anticoagulant agent warfarin, no haemorrhagic complications post-arthrocentesis were noted ${ }^{28}$.

\section{CONCLUSION}

Arthrocentesis of the temporomandibular joint is a minimally invasive method of treatment located at the boundary between conservative and surgical therapy. It is usually performed on an out-patient basis under local anaesthesia. It is used for both acute block caused by displacement of the articular disc and treatment of degenerative inflammatory joint disease. The main objective of arthrocentesis is to wash out inflammatory mediators, release the disc, break adhesions, eliminate pain and improve joint mobility.

It is a method with a minimum number of complications, it is simple and not demanding in terms of instruments, and it can be performed repeatedly. Therefore, it has become widespread and very popular in the treatment of internal disorders of the temporomandibular joint. 


\section{ACKNOWLEDGEMENT}

Authorship contributions: All authors contributed equally to preparing the manuscript.

Conflict of interest statement: None declared.

\section{REFERENCES}

1. Machoň V, Hirjak D. Manuál miniinvazivní léčby čelistního kloubu. StomaTeam 2009:22-32.

2. Aceves-Avila FJ, Delgadillo-Ruano MA, Ramos-Remus C, GómezVargas A, Gutiérrez-Ureña $S$. The first descriptions of therapeutic arthrocentesis: a historical note. Rheumatology 2003;42(1):180-3.

3. Nitzan DW, Dolwick MF, Martinez GA. Temporomandibular Joint Arthrocentesis: A Simplified Treatment for Severe, Limited Mouth Opening. J Oral maxillofac surf 1991;49:1163-7.

4. Guarda-Nardini L, Manfredini D, Ferronato G. Arthrocentesis of the temporomandibular joint: a proposal for a single-needle technique. Oral Surg Oral Med Oral Pathol Oral Radiol Endod 2008;106(4):483-6.

5. Monje-Gil F, Nitzan D, Gonzales-Garcia R. Temporomandibular join arthrocentesis. Review of the literature. Med Oral Patol Oral Cir Buca 2012;17(4),575-81. doi:10.4317/medoval.17670

6. Tozoglu S, Al- Belasy FA, Dolwick MF. A review of techniques of lysis and Lavage of the TMJ. Brit J Oral Maxillofac Surg 2011;49(4),302-9. doi:10.1016/j.bjoms.2012.03.008

7. Shinjo H, Nakata K, Shino K, Hamada M, Nakamura N, Mae T, Miyama T, Horibe S, Yoshikawa H, Ochi T. Effect of irrigation solutions for arthroscopic Sumery on intraarticular tissue: comparison in human meniskus-derived primary cell culture between lactate Ringer's solution and saline solution. J Orthop Res 2002;20:1305-10.

8. Zardeneta G, Milam SB, Schmitz JP. Elution of proteins by continuous temporomandibular joint arthrocentesis. J Oral Maxilofac Surg 1997;55:709-17.

9. Kaneyama K, Segami N, Nishimura M, Sato J, Fujimura K, Yoshimura $H$. The ideal lavage volume for removing bradykinin, interleukin- 6 , and protein from the temporomandibular joint by arthrocentesis. J Oral Maxillofac Surg 2004;62:657-61.

10. Laskin DM, Greene CS, Hylander WL, (eds.). Temporamandibular disorders-an evidence-based approach to diagnosis and treatment. Chicago Quintessence 2006;441-81.

11. Guarda-Nardini L, Manfredini D, Ferronato G. Arthrocentesis of the temporomandibular joint: a proposal for a single needle technique Oral Surg Oral Med Oral Pathol Oral Radiol Endod 2008;106:483-6.

12. Shinohara EH, Pardo-Kaba SC, Martini MZ, Horikawa FK. Single punc ture for TMJ arthrocentesis. An effective technique for hydraulic dis tention of the superior joint space. National Journal of Maxillofac Surg 2012;3:96-7.

13. Emshoff R, Puffer P, Rudisch A, Gassner R. Temporomandibular joint pain- relationship to internal derangement type, osteoarthrosis, and synovial fluid mediator level or tumor necrosis factor-alpha. Oral Surg Oral Med Oral Pathol Oral Radiol Endod 2000;90:442-9.

14. Nitzan DW, Price A The use of arthrocentesis for the treatment of osteoarthritic temporomandibular joints. J Oral Maxillofac Surg 2001;59:1154-9.

15. Manfredini D, Rancitelli D, Ferronato G, Guarda-Nardini L. Arthrocentesis with or without additional drugs in temporomandibular joint inflammatory-degenerative disease-comparison of six treatment protocols. J Oral Rehabil 2012;39(4):245-51.

16. Nishimura M, Segami N, Kaneyama K, Sato J, Fujimura K.Comparison of cytokine level in synovial fluid between successful and unsuccesfull cases in arthrocentesis of the temporomandibular joint. J Oral Maxillofacial Surg 2004;3:284-7.

17. Emshoff R, Puffer P, Strobl H, Gassner R.Effect of temporomandibular joint arthrocentesis on synovial fluid mediator level of tumor necrosis factor-alpha-implications for treatment outcome. Int J Oral Maxillofac Surg 2000;3:176-82.

18. Gulen $\mathrm{H}$, Ataoglu $\mathrm{H}$, Haliloglu S, Isik K. Proinflammatory cytokines in temporomandibular joint synovial fluid efore and after arthrocentesis. Oral Surg Oral Med Oral Pathol Oral Radiol Endod. 2009;5:e1-e4.

19. Tvrdý $P$, Heinz $P$, Hrdina L, Galandáková A, Pazdera J. Determination of cytokine levels in joint fluid as an alternative option for examination of internal Temporomandibular Joint Disorders (retrospective study) Česká stomatologie 2011;4:82-8.

20. Nitzan DW, Samson B, Better H. Long-term outcome of arthrocentesis for sudden-onset, persistent, severe closed lock of the temporomandibular joint. J Oral Maxillofac Surg 1997;55:151-7.

21. Hosaka H, Murakami K, Goto K, lizuka T. Outcome of arthrocentesis for temporomandibular joint with closed lock at 3 years follow up. Oral Surg Oral Med Oral Pathol Oral Radiol Endod 1996;82:501-4.

22. Stegenga B. Temporomandibular joint degenerative disease: clinical diagnosis. In: Stegenga B, de Bont LGM, editors. Management of temporomandibular joint degenerative diseases. Basel: Birkhäuser Verlag; 1996. p. 18-9

23. Sembronio S, Albiero A, Toro C, Robiony M, Politi M. Is there a role for arthrocentesis in recapturing the displaced disc in patiens with closed lock of the temporomandibular joint? Oral Surg Oral Med Oral Pathol Oral Radiol Endod 2008;105:274-80.

24. Rehman KU, Hall T. Single needle arthrocentesis. Br J Oral Maxillofac Surg 2009;47:403-4.

25. Rahal A, Poirier J, Ahmarani C. Single-puncture arthrocentesis - introducing a new technique and a novel device. J Oral Maxillofac Surg 2009;67:1771-3.

26. Carroll TA, Smith K, Jakubowski J. Extradural haematoma following temporomandibular joint arthrocentesis and lavage. Br J Neurosurg 2000;14:152-4.

27. Nitzan DW. Arthrocentesis-incentives for using this minimally invasive approach for temporomandibular disorders. Oral Maxillofac Surg Clin North Am 2006;18:311-28.

28. Thumboo J, O'Duffy JD. A prospective study of the safety of joint and soft tissue aspirations and injections in patients taking warfarin sodium. Arthritis Rheum 1998:41:736-9. 